\title{
VARIATION IN CLASS SIZE, THE CLASS SIZE PARADOX, AND SOME CONSEQUENCES FOR STUDENTS
}

Scott L. Feld, Department of Sociology, SUNY at Stony Brook, Stony Brook, New York, and Bernard Grofman, School of Social Sciences, University of California, Irvine, Irvine, California

Even where a total allocation of resources is fixed, it is often possible to vary the distribution of the given resources. This distribution can have important, if sometimes hidden, consequences. In the case of universities, considering the mean class size as given, three nonobvious consequences of the amount of variation in class size are examined. (1) The average class size experienced by students is directly related to the amount of variation in class size. (2) Overall student attendance is inversely related to the amount of variation in class size, (3) Overall student participation time is directly related to the amount of variation in class size. The logic of these relationships is explored, and data on 49 departments and interdisciplinary programs at one university are used to illustrate the nature and extent of the class size paradox at one university.

Key words: group size; attendance; participation; policy; classroom

At a large state university in the northeastern Unites States, students experience an average class size of 147 students. This is true despite the fact that this university has an average class size of 40 students per class. How is this possible? In this article, we show that this apparent paradox is a necessary consequence of variation in the sizes of classes where some are very large and others very small. Although this variation might appear to add nothing but variety to the experience of students, when examined more closely, the variation in class size has the consequence that only a few students at a time can experience the smaller classes while very many students can simultaneously 
experience the larger classes. The fact of variation in the sizes of classes necessarily results in students experiencing an average class size larger than the average class size for the school. We call this the class size paradox. In this article, in addition to exploring the logic of the class size paradox, we consider some of the other consequences of variation in class size for students. Others, particularly Barker and his associates (Barker and Gump, 1968) have concentrated on the consequences of increasing size. We show how the form of these relationships implies that variation in class size can lead to lower attendance of students in their classes, and in contrast, we also show how variation in class size can increase the amount of time that students are able to participate in their classes. There are many other considerations entering into these decisions including the range of specialization and types of instruction offered in courses. Our purpose is to specify some consequences of variations in class size to contribute to informed decision making by all concerned parties.

We define the class size paradox specifically as the discrepancy in the perceptions of the situation between faculty and students. The faculty experience one average class size (the average class size for the university) and the students experience another average class size. Elsewhere (Feld and Grofman, 1977), we have shown that the existence of this paradox can be the source of conflict between faculty and students where faculty interests favor greater variation in the size of classes and student interests favor more uniformity in class size. Here, we investigate the nature of the class size paradox and show how it manifests itself at one university.

In the spring of 1974 , at the State University of New York at Stony Brook, 737 classes were taught according to the published Teacher Evaluation Survey, Spring 1974. These classes had a mean of 40.5 students and a standard deviation of 65.8 (excluding 9 missing cases). Consequently, students experienced a mean class size of 147 , which is approximately 3.7 times the mean class size for the university. This is because the many students who took the large classes are averaged in with the few who took the small classes resulting in a high experienced class size. This excessively large experienced class size could easily have been reduced without any additional classes being taught or any. increase in the size of the faculty. The mean experienced class size could be reduced by $73 \%$ (to be equal to the average class size for the school) merely by redistributing the size of the classes so most of them would be the same size.

We can explicate the nature of the class size illusion by showing the general equations that determine the experienced average class sizes for faculty and students. ${ }^{1}$ The mean class size experienced by faculty is 
exactly the same as the average class size for the entire university which is:

$$
\bar{s}_{\mathrm{f}}=\sum_{\mathrm{i}=1}^{\mathrm{n}} \mathrm{s}_{\mathrm{i}} / \mathrm{n}
$$

where $\bar{s}_{f}$ is the average class size experienced by faculty, $s_{i}$ is the size of class $i$, and there are $n$ classes. This is the case because each class is experienced by exactly one faculty member, so these $n$ experiences are averaged.

However, the average class size experienced by students is different because each class of size $s_{i}$ is experienced by $s_{i}$ students and these

$$
\sum_{i=1}^{n} s_{i}
$$

experiences must be averaged. The result of this is that the average class size experienced by students is as follows:

$$
\bar{s}_{\mathrm{t}}=\sum_{\mathrm{i}=1}^{\mathrm{n}} \mathrm{s}_{\mathrm{i}} * s_{\mathrm{i}} / \sum_{\mathrm{i}=1}^{\mathrm{n}} \mathrm{s}_{\mathrm{i}}=\sum_{\mathrm{i}=1}^{\mathrm{n}} \mathrm{s}_{\mathrm{i}}^{2} / \sum_{\mathrm{i}=1}^{\mathrm{n}} \mathrm{s}_{\mathrm{i}}
$$

where $\bar{s}_{t}$ is the average class size experienced by students. We can show that this is necessarily greater than or equal to the average class size experienced by faculty by considering the generalized comparison of their experiences.

We describe the size of the class size paradox in terms of the ratio between the two perceptions. The perceptual ratio, PR, is thus defined as follows:

$$
P R=\bar{s}_{t} / s_{f}=n \sum_{i=1}^{n} s_{i}^{2} /\left(\sum_{i=1}^{n} s_{i}\right)^{2}
$$

Some slight manipulations of this expression can produce an expression for the perceptual ratio in terms of the mean, $\mu$, and variance, $\sigma^{2}$, of the sizes of classes as follows:

$$
\mathrm{PR}=1+\sigma^{2} / \mu^{2}
$$

Note that the perceptual ratio is one plus the squared coefficient of 
variation of class sizes. The inequality of class sizes is responsible for the discrepancy in perceptions. When there is no inequality of class sizes, then the perceptions of faculty and students are identical, but so long as there is any variation in class sizes, the average perception of students must be greater than that of faculty and the perceptual ratio is greater than one. In the case of SUNY, Stony Brook, it can be seen that the perceptual ratio is 3.7 and can be calculated from the standard deviation, 65.8, and the mean, 40.5, using the above equation.

Similar types of calculations can be made for each department within the university. It might be that the variation in class size would be due to the differences between departments, and within the departments classes are nearly of equal size. The class size paradox would then remain as a function of the variation in class size between departments; the large class size departments (with their large number of students) would bring up the student average more than the faculty average. Although our data do show large differences between departments, there is still a large amount of variation within each department. Initially, one might expect that the students who major in those fields with greatness variation would be those experiencing the greatest class sizes. However, the largest classes in a department are ordinarily those which are directed towards nonmajors. The result is that the experience of class size of many nonmajors is increased, and the majors can then have small classes for their more specialized courses. So each department may benefit its own majors at the expense of nonmajors. The aggregate consequence is an increase in the experienced class size of all students because all students take some of their courses outside their major fields. The mean class size experienced by all students taking classes within a department, and the mean class size experienced by the faculty in the department (and the perceptual ratios in the larger departments) are displayed in Table I for the 49 departments and interdisciplinary programs at SUNY, Stony Brook. The inequitable distribution of resources between departments is one cause of the overall class size variation. In addition, each department contributes to the variation through its decisions to have large variation in its own class sizes as evidenced by the large perceptual ratios within each department. Although minimizing variation in class size is not currently an important criterion in class offerings in most departments, it should be clear that departments can minimize variation by scheduling all classes of similar size in proportion to the student demand for each course.

According to a survey we conducted on 117 students in 3 classes, nearly all students preferred classes of size 40 to those much larger, and most students $(60 \%)$ preferred all classes of size 50 to half of size 
TABLE I. Perceptions of Average Class Size for Students and Faculty Within Each of the 49 Departments and Interdisciplinary Programs at SUNY, Stony Brook. ${ }^{a}$

\begin{tabular}{|c|c|c|c|c|}
\hline Department or program & $\begin{array}{l}\text { Faculty } \\
\text { mean }\end{array}$ & $\begin{array}{l}\text { Student } \\
\text { mean }\end{array}$ & $\begin{array}{c}\text { Perceptual ratio } \\
\text { (for departments } \\
\text { offering } 20 \text { courses } \\
\text { or more) }\end{array}$ & $\mathrm{N}$ \\
\hline Anthropology & 63 & 131 & & 17 \\
\hline Art & 34 & 99 & 2.9 & 35 \\
\hline Biology & 71 & 206 & 2.9 & 29 \\
\hline Black studies & 17 & 27 & & 13 \\
\hline Chemistry & 100 & 154 & 1.5 & 24 \\
\hline Chinese & 18 & 20 & & 3 \\
\hline Classics & 18 & 18 & & 1 \\
\hline Comparative literature & 11 & 16 & & 10 \\
\hline Economics & 48 & 96 & 2.0 & 22 \\
\hline Education & 47 & 100 & 2.1 & 29 \\
\hline English & 25 & 33 & 1.3 & 60 \\
\hline Environmental studies & 80 & 123 & & 3 \\
\hline Earth and space studies & 70 & 289 & & 15 \\
\hline Mechanics & 12 & 14 & & 8 \\
\hline Electrical sciences & 25 & 37 & & 11 \\
\hline Engineering & 57 & 64 & & 4 \\
\hline Interdept. engineering & 53 & 103 & & 5 \\
\hline Materials science & 4 & 5 & & 5 \\
\hline Foreign language teaching & 16 & 16 & & 1 \\
\hline French & 17 & 20 & 1.2 & 33 \\
\hline German & 15 & 20 & & 16 \\
\hline Greek & 6 & 6 & & 1 \\
\hline Hebrew & 12 & 14 & & 7 \\
\hline History & 43 & 65 & & 32 \\
\hline Interdisciplinary courses & 53 & 60 & & 4 \\
\hline Italian & 17 & 20 & & 16 \\
\hline Latin & 14 & 21 & & 2 \\
\hline Linguistics & 33 & 50 & & 8 \\
\hline Applied math and statistics & 54 & 83 & & 11 \\
\hline Computer science & 84 & 129 & & 6 \\
\hline Interdept. math & 24 & 25 & & 4 \\
\hline Mathematics & 28 & 44 & 1.6 & 42 \\
\hline Music & 55 & 659 & 12.0 & 30 \\
\hline Philosophy & 31 & 40 & 1.3 & 30 \\
\hline Physics & 36 & 103 & 2.9 & 23 \\
\hline Political science & 61 & 91 & 1.5 & 26 \\
\hline Portuguese & 6 & 6 & & 1 \\
\hline Puerto Rican studies & 8 & 9 & & 5 \\
\hline Polish & 11 & 11 & & 2 \\
\hline Psychology & 64 & 318 & 5.0 & 31 \\
\hline Religious studies & 27 & 27 & & 1 \\
\hline Russian & 14 & 31 & & 11 \\
\hline Sociology & 62 & 109 & 1.8 & 40 \\
\hline Spanish & 20 & 23 & & 19 \\
\hline Interdisc. social sciences & 15 & 17 & & 2 \\
\hline Swedish & 10 & 10 & & 2 \\
\hline Theatre & 23 & 43 & 1.8 & 27 \\
\hline Yiddish & 19 & 19 & & 1 \\
\hline
\end{tabular}

a It should be clear that this table provides all necessary information for any combination of departments and programs using Eqs. 1-4.

${ }^{b}$ The variation in class size is necessarily limited when few classes are given. Students would not be likely to take as many courses within any of these smaller programs. 
30 and half of size 150 , despite the fact that their experience is much more like the more varied distribution of classes. In addition, practically no students preferred classes of size 10 or smaller to somewhat larger classes ( 20 or 30 ). The actual distribution of classes includes many classes smaller than that, which necessitates many more undesirable large classes. So based upon these preferences, a decrease in class size variation could increase the satisfaction of students.

Now, we can consider some of the behavioral consequences of variation in the size of classes. Elsewhere (Feld and Grofman, 1976), we have shown that many theories lead us to expect the finding in our SUNY, Stony Brook data that students are less likely to attend larger classes than smaller classes. If a large proportion of a small class attends, it means a few people are attending class, but if a small proportion of a large class attend, it means that many people are staying home. In the data from the Teacher Evaluation Survey, at the end of the year when the survey was made, classes had an average attendance of $62 \%$ over all classes, but only $52 \%$ of the students who were registered for classes were attending class. Classes of an average size, 35 to 45 , had $62 \%$ attendance on the average, but smaller classes had higher attendance and larger classes had smaller attendance with the result being lower average attendance overall. If we can assume that class size is the cause of the attendance differences (as appears likely but as cannot be definitively shown without experiments), then we can predict that if all classes were of similar size around the average, the attendance of students would increase by $20 \%$. This is a substantial increase in student attendance, and even if the increase is only a part of this, it is an important consideration, insofar as we believe that students benefit from attending class.

Finally, we consider a behavioral consequence with opposite implications. Becker et al. (1973), working with the theoretical perspective of ecological psychology, found that small classes (under 20 students) devoted more than twice the amount of class time to student participation than either medium $(21-50)$ or large $(50+)$ classes. ${ }^{2}$ Although this finding is not statistically significant with their small sample size, it is consistent with the differences in format that would be expected. Small classes are more likely to be run as seminars or discussions, and large classes are more likely to be run as lectures. To demonstrate this, we calculated the proportion of classes of each size using each format using information on the actual format that was used provided by the instructors in the Teacher Evaluation Survey. Excluding all lab and other formats, and liberally counting all lecture-discussion formats as half and half, we found that $52 \%$ 
$(\mathrm{N}=250.5)$ of the small classes (under 20 ), were conducted as discussions, compared with $35 \%(\mathrm{~N}=226)$ of the medium classes $(21-50)$ and $19 \%(\mathrm{~N}=118.5)$ of the large classes $(51+)$. So if format is the major determinant of time spent on student participation, then it is very clear that larger classes spend less time on student participation than small ones. If we wish to maximize the average time students get to participate in classes, we should maximize the amount of time classes spend on student participation all together. Since, there is a minimum time that a class can spend on participation, $0 \%$, if we have one or a few enormous classes having no participation time at all and all the rest, being small, having maximum participation time, the total time spent on student participation is maximized, and therefore the average time spent on participation is maximized. The total participation time is then the number of small classes (nearly all classes) times the amount of time spent on participation in these classes. The total number of hours that all students spend in class is divided into this to produce the average time per class per student. So by increasing the number of small classes while having a few extraordinarily large classes, the amount of participation time per student could be substantially increased. This implies that the participation time available to students increases with the variance in class sizes.

In summary, we have identified three important consequences of variation in class size for students that have heretofore been neglected. (1) Large variation means that students experience many more of the less desired large classes than the more desired smaller (medium-sized) classes. (2) Large variation in classes combined with the fact that students are less likely to attend the larger classes lead to a lower overall attendance in classes by students. (3) Large variation in class size including many of the smallest classes, combined with the fact that smaller classes devote more time to student participation, leads to larger amount of average student participation time in class.

We have drawn out these consequences so as to clarify these particular impacts of variation. We hope that these will be taken into account along with other considerations of policy makers.

\section{ACKNOWLEDGMENTS}

We would like to thank Paul Allison, Kenneth Feldman, and John Logan for helpful criticisms and suggestions on earlier drafts of this paper. 


\section{FOOTNOTES}

${ }^{1}$ The following calculations are under the assumption that each student takes exactly one class, and each instructor teaches exactly one class. However, it can be easily shown that the results are identical if all students take any fixed number of classes, and all instructors teach any fixed number of classes, because each of their individual experiences has equal weight in the overall average. Where there are variable course loads, each individual experience of those with few experiences weighs more heavily in the overall average. However, the results are similar so long as there is no substantial correlation between the average course size of students and instructors and their course load. Where there is some substantial correlation, slightly modified calculations can be made.

${ }^{2}$ Note that they also found that the absolute number of students who participate is fairly constant in classes of all sizes, indicating that frequency of student participation is independent of variation in class size.

\section{REFERENCES}

Barker, R. G., and Gump, P. V. (1964). Big School Small School: High School Size and Student Behavior, Stanford University Press.

Becker, F. D., Sommer, R., Bee, J., Oxley, B. (1973). College Classroom Ecology. Sociometry 514-525.

Feld, S. L., and Grofman, B. (1976). College Class Size and Voluntary Attendance: An Effect of Group Size. Unpublished manuscript.

Feld, S. L., and Grofman, B. (1977). A Note on the Class Size Paradox and Conflict of Interest Among and Between Students and Faculty. Unpublished manuscript.

Teacher Evaluation, Spring 1974, State University of New York at Stony Brook. 